\title{
Late avulsion of a free flap in a patient with severe psychiatric illness: Establishing a successful salvage strategy
}

\author{
Clara Schaffer ${ }^{1}$, Andrew Hart ${ }^{2}$, William Watfa ${ }^{1}$, Wassim Raffoul ${ }^{1}$, Pietro Giovanni di Summa ${ }^{1,2}$ \\ ${ }^{1}$ Department of Plastic, Reconstructive and Hand Surgery, University Hospital of Lausanne (CHUV), Lausanne, Switzerland; ${ }^{2}$ Canniesburn \\ Plastic Surgery Unit, Glasgow Royal Infirmary, Glasgow, UK
}

Post-traumatic defects of the distal third of the leg often require skipping a few steps of the well-established reconstructive ladder, due to the limited local reliable reconstructive options. In rare cases, the reconstructive plan and flap choice may encounter challenges when the patient has psychiatric illness affecting compliance with postoperative care. We describe a case of a patient with severe intellectual disability and an open fracture of the distal lower limb. After fracture management and debridement of devitalized tissues, the resultant soft tissue defect was covered with a free gracilis flap. On postoperative day 7, the patient ripped out the newly transplanted flap. The flap was too traumatized for salvage, so a contralateral free gracilis muscle flap was used. The patient showed good aesthetic and functional outcomes at a 1-year follow-up. When planning the postoperative management of patients with psychiatric illness, less complex and more robust procedures may be preferred over a long and complex surgical reconstruction requiring good compliance with postoperative care. The medical team should be aware of the risk of postoperative collapse, focus on the prevention of pain, and be wary of drug interactions. Whenever necessary, free tissue transfer should be performed despite potential compliance issues.

Keywords Free tissue flap / Lower extremity / Mental disorder / Reconstruction

\author{
Correspondence: \\ Pietro Giovanni di Summa \\ Department of Plastic, Reconstructive \\ and Hand Surgery, University Hospital \\ of Lausanne (CHUV), Rue du Bugnon \\ 46, Lausanne 1005, Switzerland \\ Tel: +41-21-314-2525 \\ Fax: +41-21-314-2530 \\ E-mail: pietro.di-summa@chuv.ch
}

This article was presented at the European Federation Societies of Microsurgery Congress on May 5-8, 2018, in Belgrade, Serbia.

Received: July 27, $2018 \bullet$ Revised: January 14, $2019 \bullet$ Accepted: February 1, 2019

pISSN: 2234-6163 • elSSN: 2234-6171 • https://doi.org/10.5999/aps.2018.01039 • Arch Plast Surg 2019;46:589-593

\section{INTRODUCTION}

Post-traumatic lower limb reconstruction represents a challenge in plastic surgery. The initial management includes extensive debridement of devitalized tissue, as well as reduction and stabilization of fractures if needed. Flap reconstruction is commonly required, with options including a local/propeller flap, a pedicled flap, or a free flap. However, defects of the distal third of the leg often require skipping a few steps of the well-established re- constructive ladder. This is due to the limited local reliable reconstructive options for this area, leading to a lower threshold for free tissue transfer [1]. The most commonly used muscular free flaps for the distal limb include gracilis and latissimus dorsi myocutaneous or muscle flaps, which can potentially cover defects of all sizes. Among the variety of perforator flaps, the anterolateral thigh flap probably represents the most versatile option due to potential uses of its multiple components. The choice of a flap depends on the defect characteristics, the recon- 
structive needs (function, sensitivity, and aesthetics), and the experience of the surgeon.

However, the reconstructive plan and flap choice may encounter challenges if the patient has a psychiatric disorder. Severe intellectual disability is associated with twice as many complications after surgery as in the general population [2]. However, little is known about the postoperative course of patients with learning disabilities or psychiatric disorders after reconstructive surgery procedures, particularly free flap surgery. Guidelines for the pre- and postoperative management of these patients are lacking. The literature contains case reports of patients suffering from psychosis, schizophrenia, and other neuropsychological disorders who required free flap surgery, mainly following hand self-amputation [3]. Other studies have focused on the postoperative course of patients experiencing alcohol withdrawal syndrome after oncologic surgery [4-6]. Few cases of microsurgical free tissue transfer in patients with intellectual disability have described [7].

In this report, we present our experience with a patient suffering from intellectual disability, who presented with an open fracture of the distal third of the leg, requiring fracture stabilization followed by soft tissue coverage with a contralateral free gracilis muscle flap. The postoperative self-inflicted flap avulsion due to psychiatric deterioration required emergent hemostasis and a secondary reconstructive procedure, using a free gracilis muscle flap harvested from the right leg. The patient's postoperative course was uneventful, and the secondary procedure resulted in successful long-term coverage.

\section{CASE}

A 48-year-old male patient with known severe autism and learning disability with psychotic traits, living in a care institution, fell out of the window of his room from a 3-m height, apparently without suicidal intent. He was found with a Gustilo-Anderson IIIB fracture on the right tibia. After antibiotic prophylaxis with intravenous amoxicillin and clavulanic acid, emergency reduction and stabilization of the fracture with a tibiocalcaneal external fixator (Orthofix; Orthofix Holdings Inc., Bussolengo, Italy) was performed. Unfortunately, the patient developed cutaneous necrosis of the surgical site, which required repeated surgical debridement and replacement of the external fixator material on postoperative days (POD) 9, 11, and 21. The last procedure allowed definitive tibial osteosynthesis. The resultant pyramidshaped soft tissue defect $(8 \times 14 \mathrm{~cm})$ was covered with a left free gracilis muscle flap, and anastomosed in a termino-lateral manner to the posterior tibial artery and in a termino-terminal manner with one of the posterior tibial venae comitantes. The mus-
Fig. 1. Intraoperative images of the first reconstruction procedure

(A) A free gracilis muscle flap harvested from the left thigh and the prepared posterior tibial recipient vessels (green arrows). (B) The flap was anastomosed in a termino-lateral manner to the posterior tibial artery (red arrow) and in a termino-terminal manner with one of the posterior tibial venae comitantes (blue arrow). (C) In setting of the muscular flap before split-thickness skin graft coverage.
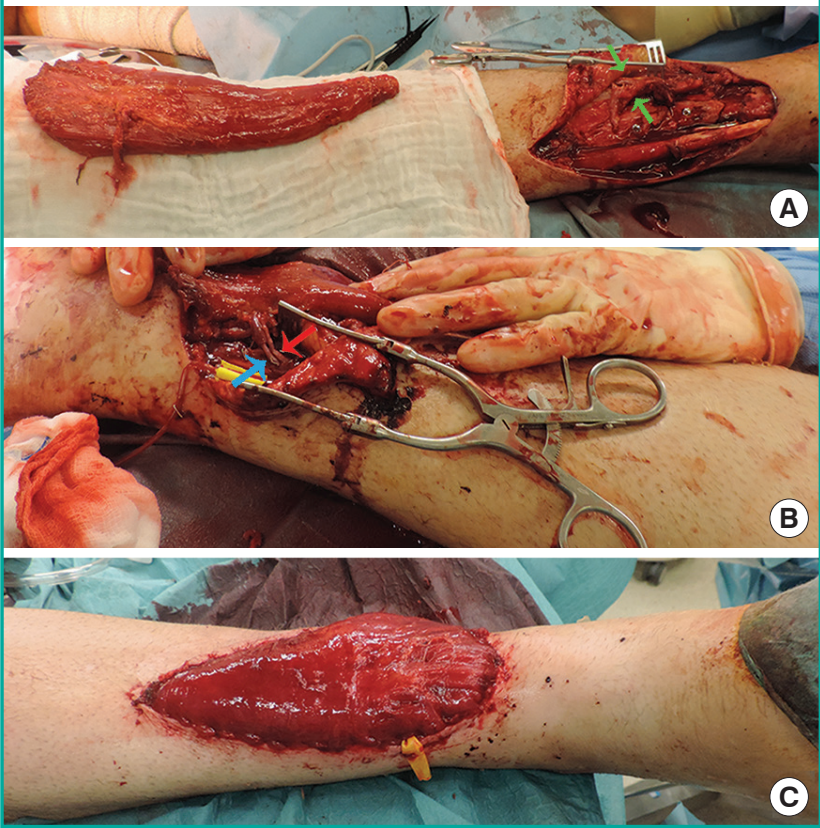

cle flap was then covered with a split-thickness skin graft (Fig. 1). The flap was monitored hourly during the first 24 hours, and strict bed rest with slight limb elevation was prescribed. Surveillance intervals were progressively increased on POD2 and POD3 to four times/day. Despite the difficulty in adequately communicating flap care needs to the patient, he complied with splint immobilization and bedrest for the first 6 days.

On POD7, during the night, the patient removed the splint and ripped out the newly transplanted flap from his right leg, without a specific reason, with consequent bleeding from the posterior tibial artery at the level of the anastomosis, requiring emergency hemostasis overnight. Due to severe damage to the flap, it was impossible to re-anastomose (Fig. 2), and the defect was temporarily covered with Epigard (Biovision, Ilmenau, Germany). Postoperatively, the patient remained sedated and placed under continuous surveillance in the intermediate care unit. On the next day, operatory wound revision revealed the posterior tibial artery to be thrombosed but continous, and a second posterior tibial vena comitans was intact. After resecting the thrombosed and traumatized area of the artery, a second free gracilis muscle flap was raised and anastomosed in a termino-terminal manner to the posterior tibial artery and residual vena comitans. On this occasion, the flap was protected with a 
Fig. 2. Self-inflicted injury to the free gracilis flap

(A) Bedside wound status after the flap was ripped out by the patient. (B) A severely damaged flap that was impossible to re-anastomose.

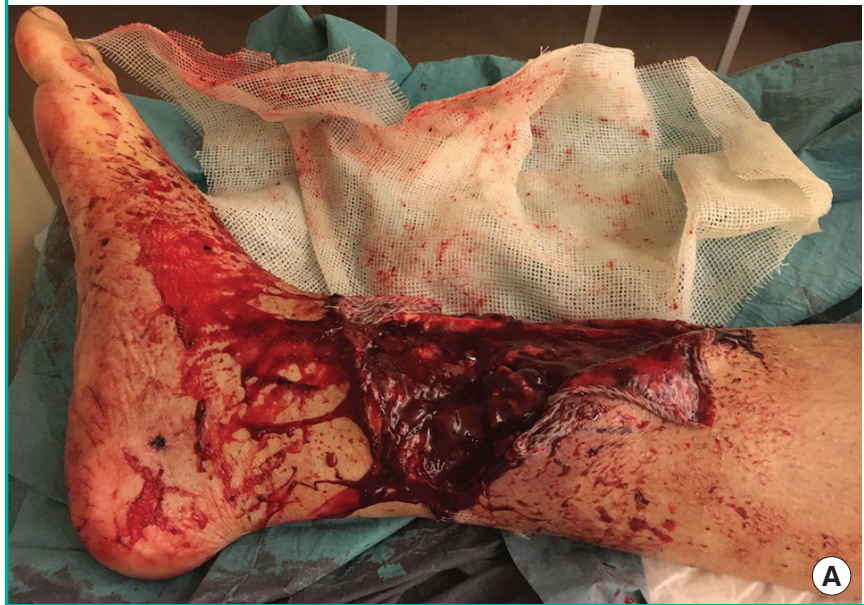

full-leg above-knee cast. He was again placed on bed rest, with a 24-hour nursing team surveillance. A psychiatric nurse and physician regularly visited the patient and counselled the medical team to adjust his antipsychotic medications (olanzapine and topiramate) and tranquilizer (lorazepam). The doses remained unchanged, but haloperidol and clomethiazole were added to prevent new acute schizophrenic episodes. Analgesic treatment, combining long-acting and short-acting oxycodone, was administered according to the level of pain, as assessed by the Faces Pain Scale. The patient did not develop further medical, flap-related, or psychiatric complications, and remained compliant for the rest of the hospital stay. He was transferred to his psychiatric institution 13 days after the last intervention. After 6 weeks, after radiography confirmed that adequate healing had occurred mobilization (partial load) was started with a physiotherapist. Full loading was allowed after 3 months.

The patient healed uneventfully, showing good aesthetic and functional outcomes at a 1-year follow-up (Fig. 3), with preserved mobility and no donor site complications.

The patient's legal guardian provided informed consent, including permission for photographic and video documentation.

\section{DISCUSSION}

Patients with severe psychiatric conditions or learning disabilities are particularly vulnerable in the postoperative period. A previous study reported the outcomes of 38 patients with neuropsychological impairment and hand and facial traumatic injuries requiring free flap reconstruction, who underwent $40 \mathrm{mi}-$ crosurgical reconstructive procedures [7]; however, only three of those patients had severe psychiatric illness. The overall success rate $(87.5 \%)$ was similar to that of the population at large.

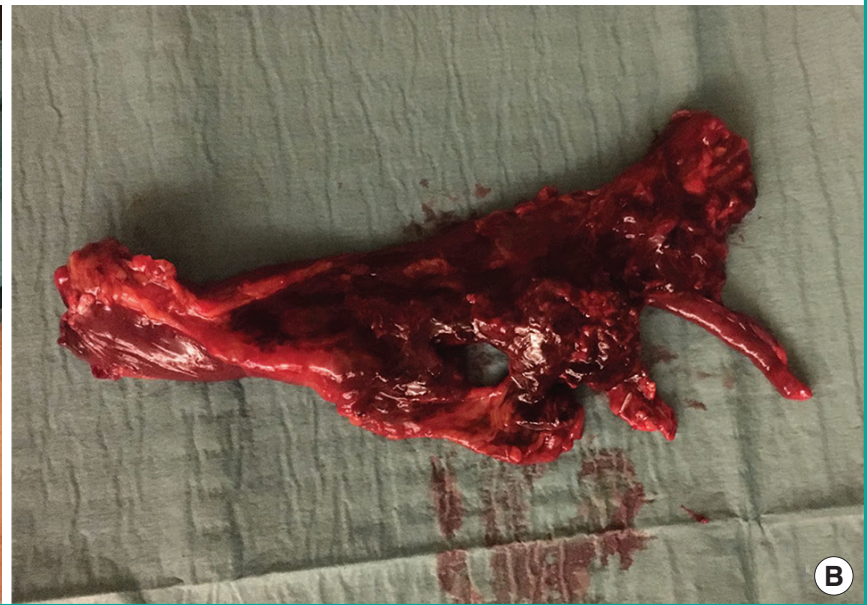

Fig. 3. Aesthetic outcomes at a 1-year follow-up

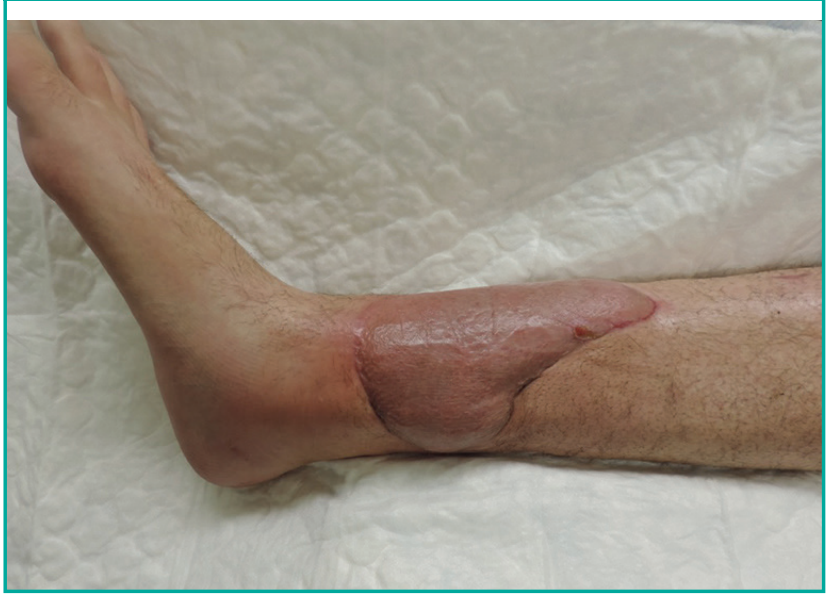

The reported relapse rate of the psychiatric disorders was $23.1 \%$, but the flap-related complication and re-intervention rates were not stated. The main issue during hospitalization was poor compliance with postoperative immobilization. However, all flaps healed uneventfully. Psychiatric patients, and those with learning disabilities, often present with an altered perception of pain combined with a reduced ability to communicate symptoms [3]. Moreover, studies have raised awareness about interactions between antipsychotic, anesthetic, and analgesic medications due to enzymatic induction. In our case, even though the heavy daily antipsychotic treatment received by the patient (topiramate, olanzapine, and lorazepam) was never interrupted during the hospital stay, pharmacologic interactions between analgesic (oxycodone) and antipsychotic drugs may have decreased the effectiveness of both analgesics and neuroleptics. This phenomenon probably triggered pain or discomfort, along with psychiatric collapse, leading the patient to avulse his free 
flap in response.

When planning the postoperative management of patients with severe psychiatric illness, a learning disability, or inability to communicate, the medical team should be aware of the risk of postoperative deterioration, especially after trauma, emergency procedures, or repeated general anesthesia. Reconstruction should not be withheld, especially if soft tissue coverage of fractures or prosthetic material is needed, but postoperative compliance and patient understanding should be factored into the selection of the reconstructive technique, even in the emergency setting, and the care plan and choice of dressings. If possible, single-stage, more robust procedures, such as local flaps, should be selected over complex surgical reconstructions requiring compliance with prolonged postoperative care regimens. Locoregional anesthesia and sedation should be considered instead of general anesthesia. Nonetheless, free tissue transfer should be performed when necessary, but postoperative care should be adjusted to increase flap protection and to minimize the opportunity for self-harm.

The team should focus on optimal pain management and preventing psychiatric deterioration, as well as closely observing for early signs of decompensation (restlessness, algetic face, apathy, and muteness). Daily antipsychotic medications must be continued with advice from a psychiatrist either to adapt the dose or to add other drugs, as in our case. A pharmacologist should provide counselling regarding drug interactions and dosage adjustment on a case-by-case basis. Periodic dosage of medications may be helpful for adapting the dosage, especially for olanzapine. Despite the risk of drug interactions, optimal analgesia is essential to maintain a steady psychiatric state. Pain control can be achieved by detecting any signs of discomfort using the Faces Pain Scale, rather than the usual self-reported pain level, and combining short- and long-acting analgesics. Restricting access to the flap with a full-leg cast may prevent injuries to the flap if psychiatric collapse occurs. A multidisciplinary team comprising psychiatric, surgical, pharmacology teams, including both physicians and nurses, is most suitable for managing communication issues and promoting adherence to postoperative recommendations [8].

If acute decompensation occurs despite preventive measures, rapid neuroleptic treatment should be administered and sedation (e.g., haloperidol) should be introduced, followed by continuous monitoring.

Before discharge, the patient's medical prescriptions must be checked accurately. In this case, the oral antibiotic (rifampicin), a powerful inducer of cytochromes (CYP 2C9, 2C19, 2C8, and 3A4), was implicated in the inactivation of various drugs, potentially reducing the efficacy of antipsychotic and antalgic medica- tions. Concomitant administration is not contraindicated, but close monitoring is warranted. Close biological follow-up (with a complete blood count, hepatic function, and renal function tested at least once a week) is advised. When the analgesic medication is discontinued, the doses of antipsychotic medications should be reduced according to preoperative standards, and the added drugs may be removed progressively. Postoperative surgical and psychiatric follow-up must be scheduled before discharge. Caregivers should be highly vigilant in recognizing signs of a recurrent delusional episode [9]. A rapid return to the patient's usual surroundings and care structure is beneficial, suggesting that over-hospitalization may be deleterious rather than cautious.

On a more general level, outcomes after head and neck oncologic surgery and microsurgical tissue transfer have improved due to standardized care protocols and the early detection and treatment of patients presenting with postoperative alcohol withdrawal syndrome [10]. Patients with neuropsychological impairment should benefit from similar protocols. Further cases should be reported in order to assess flap-related and psychiatric outcomes in this particular population, and ultimately to provide clear preoperative management guidelines.

\section{NOTES}

\section{Conflict of interest}

No potential conflict of interest relevant to this article was reported.

\section{Ethical approval}

The study was approved by the Institutional Review Board of Lausanne University Hospital and performed in accordance with the principles of the Declaration of Helsinki. Written informed consent was obtained.

\section{Patient consent}

Patient's legal guardian provided written informed consent for the publication and the use of his images.

\section{Author contribution}

Study concept and design: Watfa W, di Summa PG. Data acquisition: Schaffer C. Data analysis and interpretation: Schaffer C. Drafting of the manuscript: Schaffer C. Critical revision of the manuscript for important intellectual content: Hart A, Watfa W, di Summa PG, Raffoul W. Approval of final manuscript: all authors. 


\section{ORCID}

Clara Schaffer https://orcid.org/0000-0001-7230-4984

William Watfa https://orcid.org/0000-0002-5951-9443

Wassim Raffoul https://orcid.org/0000-0002-3524-0077

\section{REFERENCES}

1. Bennett N, Choudhary S. Why climb a ladder when you can take the elevator? Plast Reconstr Surg 2000;105:2266.

2. Ailey SH, Johnson TJ, Fogg L, et al. Factors related to complications among adult patients with intellectual disabilities hospitalized at an academic medical center. Intellect Dev Disabil 2015;53:114-9.

3. Copeland LA, Zeber JE, Pugh MJ, et al. Postoperative complications in the seriously mentally ill: a systematic review of the literature. Ann Surg 2008;248:31-8.

4. Chang CC, Kao HK, Huang JJ, et al. Postoperative alcohol withdrawal syndrome and neuropsychological disorder in patients after head and neck cancer ablation followed by microsurgical free tissue transfer. J Reconstr Microsurg 2013;
29:131-6.

5. Kuo YR, Jeng SF, Lin KM, et al. Microsurgical tissue transfers for head and neck reconstruction in patients with alcohol-induced mental disorder. Ann Surg Oncol 2008;15:371-7.

6. Ferrari S, Copelli C, Bianchi B, et al. Free flaps in elderly patients: outcomes and complications in head and neck reconstruction after oncological resection. J Craniomaxillofac Surg 2013;41:167-71.

7. Lin CH, Wei FC, Chen CT, et al. Microsurgical tissue transplantation or replantation in patients with psychoneurological impairment. Plast Reconstr Surg 2001;108:1211-7.

8. Abrams TE, Vaughan-Sarrazin M, Rosenthal GE. Influence of psychiatric comorbidity on surgical mortality. Arch Surg 2010;145:947-53.

9. Strain JJ, DeMuth GW. Care of the psychotic self-amputee undergoing replantation. Ann Surg 1983;197:210-4.

10. Lansford CD, Guerriero $\mathrm{CH}$, Kocan MJ, et al. Improved outcomes in patients with head and neck cancer using a standardized care protocol for postoperative alcohol withdrawal. Arch Otolaryngol Head Neck Surg 2008;134:865-72. 